\title{
Vision-Based UAV Distribution Line Inspection Using Deep Learning
}

\section{Xuruoyan Chen}

China Jiliang University

Jiedong Zhuang

China Jiliang University

\section{Haijie Wang}

China Jiliang University

\section{Min Xie}

China Jiliang University

Enhui Zheng ( $\square$ ehzheng@cjlu.edu.cn )

China Jiliang University

\section{Research}

Keywords: Power line inspection, Vision-based inspection, Deep learning, Segmentation, UAV images

Posted Date: January 17th, 2022

DOI: https://doi.org/10.21203/rs.3.rs-1205195/v1

License: (c) (i) This work is licensed under a Creative Commons Attribution 4.0 International License. Read Full License 
Vision-based UAV distribution line inspection using deep learning

Xuruoyan Chen ${ }^{1}$, Jiedong Zhuang ${ }^{1}$, Haijie Wang ${ }^{1}$, Min Xie ${ }^{1}$, Enhui Zheng ${ }^{1,2, *}$

${ }^{*}$ Correspondence: ehzheng@cjlu. edu. cn

1 Unmanned System Application Technology Research Institute, China Jiliang University, Hangzhou 310018, China

${ }^{2}$ Hangzhou Mission Intelligent Technology Company Limited, Hangzhou 310018, China

Abstract

Regular inspection of distribution line is an important link to maintain the normal operation of 
distribution network. Using unmanned aerial vehicle (UAV) instead of manpower can save the cost of inspection. With the universal application of vision sensor in UAV and the rapid development of deep learning, Convolutional Neural Networks (CNN) is applied to the detection of power line in UAV visible images. In view of the lack of application environment inspection methods for distribution line, a vision-based UAV distribution line inspection method using deep learning and a dataset for the deep learning method of distribution line inspection task are proposed in this paper. The method proposed predicts distribution line area through the encoder-decoder structure network firstly. Image processing operation and sampling clustering are used to remove the interference. Finally, the UAV tracking direction of distribution power line is calculated according to the detected distribution line. The method can reach an inspection speed of nearly $77 \mathrm{~ms}$ per frame, the range of heading deviation error can reach $\left(-1.52^{\circ}, 1.36^{\circ}\right)$, the tracking rate nearly $100 \%$. Through the test of network and inspection method on dataset, the results show that the method proposed in this paper can be effectively, quickly and accurately applied to UAV distribution line inspection.

Keywords: Power line inspection, Vision-based inspection, Deep learning, Segmentation, UAV images

\section{Introduction}

With the rapid development of power system scale, a large number of power line have been put into operation. Distribution network is the link between the power plants and the points of consumption, occupies a considerable proportion. Power failure will cause serious economic losses and bring unexpected consequences $[1,2]$. Therefore, the distribution line needs to be inspected and maintained to ensure its normal operation.

Regular visual inspection of distribution power line is one of the most important part to maintain the normal operation of distribution network [3]. The traditional methodologies for inspecting power lines include field surveys and airborne surveys. Manual inspection is inefficient and potentially dangerous [4]. Some scenes are inaccessible to humans, e.g. a dam on a river in a tricky, or power grid in thick forests [5]. An emerging option out of all the airborne surveys is to employ a UAV [6]. High resolution images can be obtained by a small UAV that could be used in line inspections and many other applications [7]. The cost of using UAV for inspection incurs approximately one third of that of using helicopter for inspection [8-10]. Also, it is possible to overcome the low efficiency and safety problems of manual inspection [11]. In addition, it can observe the transmission line more closely and obtain more detailed images of power components, thus greatly improving the inspection accuracy.

As a lot of research on vision technology have been made and the advantage of vision sensors, the realization of UAV power line inspection based on vision has become the research hotspots of power grid inspection [12]. The most basic but the most important problem in the vision-based UAV power line inspection is the power line detection in the visible light image. Traditional image processing methods and deep learning methods are mainly vision-based power line detection methods. The use of traditional image processing methods to detect power lines developed earlier. In [13] Li al et. proposed to filter the background with a filter based on Pulse Coupled Neural Network, then use the Hough transform to detect straight lines. Finally, the results are refined by K-means clustering. Song al et. proposed to detect line segments by morphological filtering an edge map image in the local criterion and group the line segments into whole power line in the global criterion. In [14] proposed a novel 
object-based Markov random field with anisotropic weighted penalty method to distinguish the power line segments. And an envelope-based piecewise fitting method to fit the power line. With the rapid development of deep learning and the disadvantages of versatility and stability of traditional image processing methods [15-17]. Deep learning methods are gradually being used to detect power line $[2,18,19]$. Hui al et. achieve autonomous navigation of drones based on transmission towers by using faster R-CNN [20] deep learning framework to detect tower and fully convolutional network (FCN) [21] to detect power line [22]. Saurav al et. use a nested U-Net segmentation architecture to automatic autonomous visual power line segmentation [23]. Currently, the distribution line detection by using deep learning lacks the comprehensive consideration of detection speed and accuracy in application. And there is almost no public dataset for deep learning distribution line detection.

In this article, a vision-based UAV distribution line inspection method using deep learning is proposed. The main contributions are as follows: 1) A dataset for deep learning method of testing distribution line detection task is proposed. 2) A visual based inspection method for UAV distribution line is proposed. 3) Deep learning is used to segment the power line in UAV aerial images, and compared with the existing methods, the detection accuracy and speed are significantly improved.

\section{Method}

An overview of the method we adopted in the whole work is shown in the Fig. 1. The visible image of distribution line captured by UAV is input into the deep learning network to obtain the prediction results of the power line area in the image. Through the steps of image processing, sampling clustering and deviation calculation of the prediction results, the heading deviation and roll deviation for controlling the UAV line patrol are obtained.

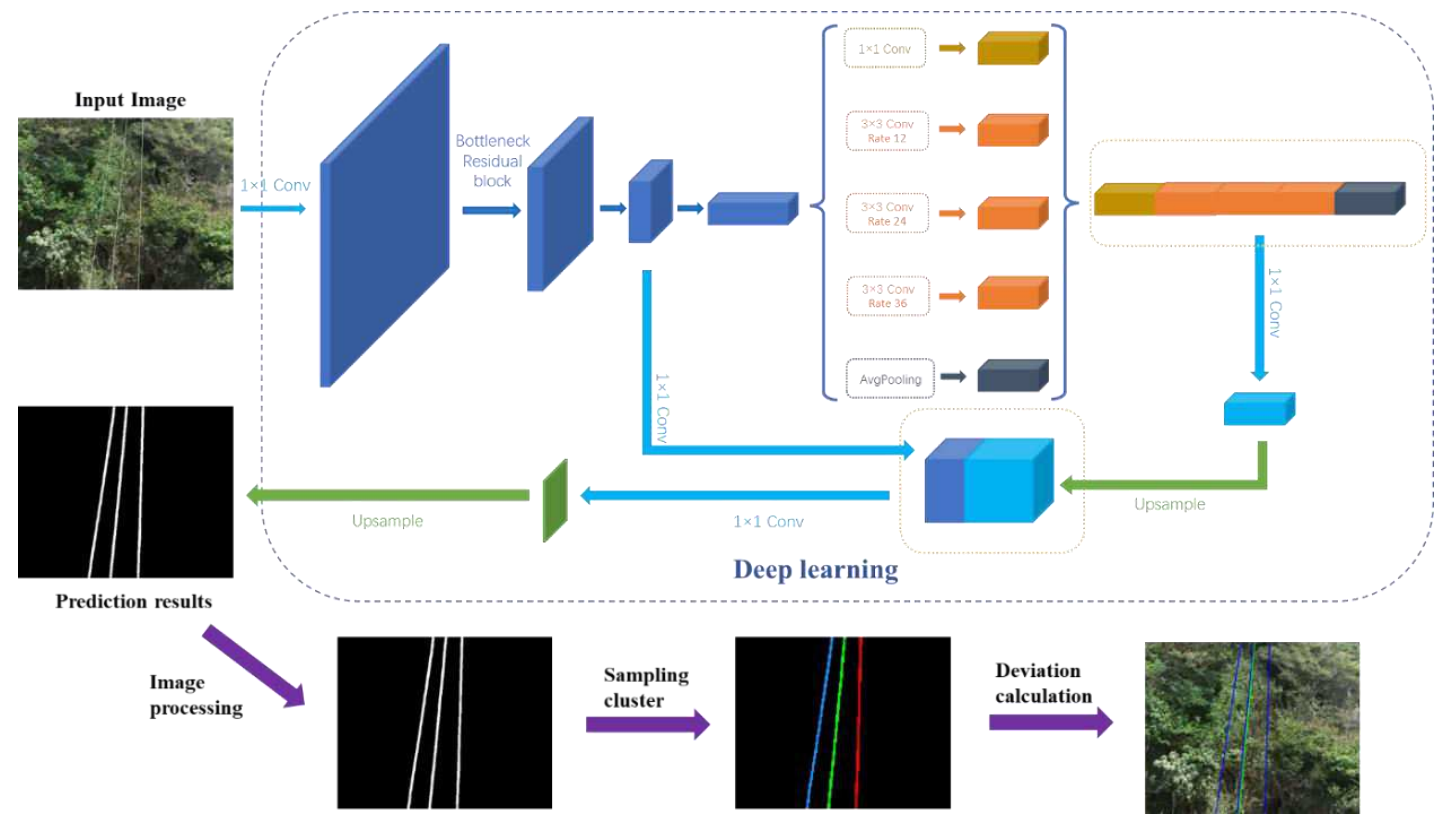

Fig.1 The overview of the distribution line inspection method.

\section{1 Model Architecture}

The advanced light-weight network model is used for semantic segmentation. The whole network adopts encoder-decoder structure. MobileNetV2 [24] and atrous spatial pyramid pooling (ASPP) [25] as an encoder module to capture contextual information, which is suitable for mobile devices. A simple 
and effective structure as decoder module to refine the segmentation results along object boundaries.

The architecture in the encoder module is MobileNetV2, which is specifically tailored for mobile and resource constrained environments. This network pushes the state of the art for mobile tailored computer vision models, by significantly decreasing the number of operations and memory needed while retaining the same accuracy. The basic idea of Depthwise Separable Convolutions is to replace a full convolutional layer with a depthwise convolutional layer and a pointwise convolutional layer. The depthwise convolutional layer performs filtering by applying a single convolutional filter per input channel. The pointwise convolutional layer responsible for building new features through $1 \times 1$ convolutional kernel computes linear combinations of the input channels. Bottleneck Residual block is used as the basic building block in the network to achieve a balance between feature extraction and computing speed. Fig. 2 the structure of the network block is a bottleneck depth-separable convolution with residuals. Firstly, the basic building block extracts the feature after expanding the dimension of input. Then, compresses the dimension and connects with the input. Informally, the set of layer activations forms a "manifold of interest". The linear bottleneck is used in the basic building block to capture the manifold of interest to prevent nonlinearities from destroying too much information. The architecture of encoder module contains the initial fully convolutional layer, 13 residual bottleneck layers and ASPP module. The output stride is defined as the ratio of input image spatial resolution to the final output spatial resolution. The model adopts output stride $=8$ by removing the last set of residual bottleneck layer operators with striding and the following operators in the MobileNetV2. The output of the last residual bottleneck layer is used as the input of ASPP module. The output of the ASPP module is the final output of encoder. The encoder output feature map contains 64 channels and rich feature information.

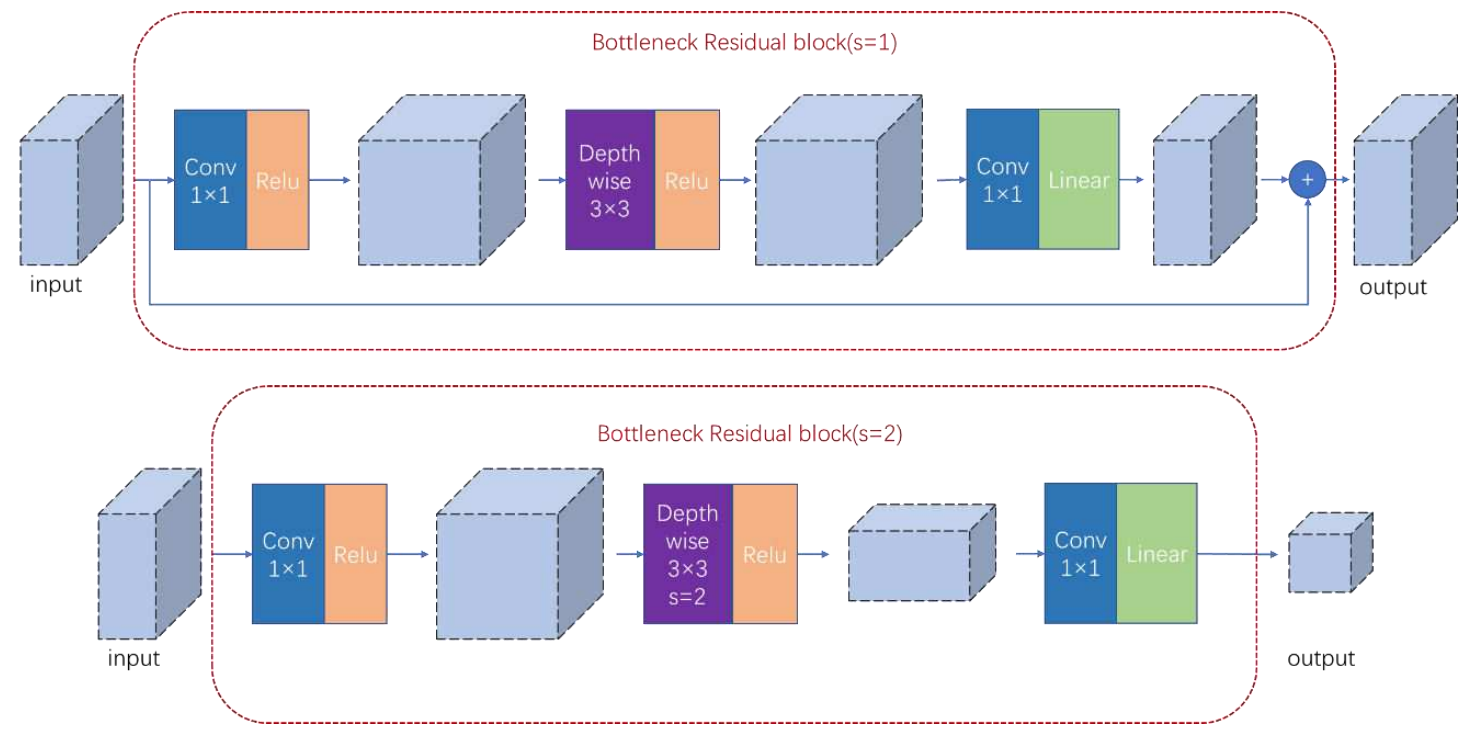

Fig.2 The structure of Bottleneck Residual block.

The structure of decoder module is similar to the decoder module which is proposed by DeepLabv3+ [25]. This simple yet effective decoder module can successfully recover object segmentation details. The output features from encoder module are computed with output stride $=8$. Firstly, the bilinear upsampling with a factor of 2 is performed on the output feature map of the encoder module, and then concatenate it with the low-level features from the encoder module that have the same spatial resolution. The deep information with low spatial resolution after multiple downsampling provides the context semantic information. The shallow information provides more precise segmentation 
information. The segmentation of target object will be more precise and accurate by concatenating the shallow information with the deep information. Different from the decoder module of DeepLabv3+, the number of channels for low-level features is not reduced but expanded. Because of the low-level features which is connected in the network only contains 24 channels, will not outweigh the importance of the output features from encoder module. Besides, it is not conducive to refine the segmentation results along object boundaries through the low-level features with too few channels. Then, the concatenated features are applied a few convolutions with a $3 \times 3$ convolutional kernel before the bilinear upsampling with a factor of 4 is performed. Finally, the features are mapped to the desired class by a $1 \times 1$ convolution layer. The output of the decoder module is also the output of the whole network, which shows the class of each pixel.

\section{2 Image Processing}

Simple but effective image processing operation is adopted for the prediction results from network to ensure the efficiency and improve the accuracy of results. The adjacent regions of the prediction results from network are connected by using morphological close operation. The morphological close operation is to dilate the image I firstly as:

$$
D[u, v]=\max _{(i, j) \in \text { kernel }} I[u+i, v+j]
$$

where $[u, v]$ stand for the pixel coordinate of image, and $[i, j]$ stand for the coordinate of kernel. Then, erode the dilated image $\mathrm{D}$ as:

$$
E[u, v]=\min _{(i, j) \in k e r n e l} D[u+i, v+j]
$$

The prediction regions of the same power line in the prediction results are connected, and the edge of the prediction area is smoothed without significantly change. Although the larger kernel of the closed operation, the more connected the whole image is, we hope that the prediction region belonging to the same power line in one connected region. It is not conducive to cluster the power line if connecting the prediction regions that are not belong to the same power line. Therefore, it is not proponent to select the kernel with a large size. In the experiment, the size of kernel is selected by $9 \times 9$.

\section{3 Sampling Cluster}

It will obtain the mask of the power line by performing semantic segmentation on the image. But there is no instance segmentation for power line, so the pixels belonging to power line in the prediction result have the same label. Density based spatial clustering of applications with noise (DBSCAN) [26] algorithm is used to cluster the set of power line pixel coordinates. The pixel coordinates of each category in the clustering result belong to the same power line. DBSCAN clustering algorithm is based on the density of data elements to identify clusters of arbitrary shape in the data. It thinks that the data close to each other often belong to the same class. Moreover, DBSCAN clustering algorithm can determine which data should be classified as noise. Because of the complexity of distribution network lines, the number of power lines in each frame of UAV real-time image is uncertain. In order to reduce the impact of clustering algorithm on the processing speed of the system, the image is downsampled, and the power line pixel coordinates are extracted for DBSCAN algorithm clustering. Because the power line occupies less pixels in the image, the sampling rate should not be too large, so the downsampling rate of 2-3 times is suggested. In the experiment, 3 times lower sampling rate is chosen. The minimum circumscribed rectangle of the coordinate set of each class of pixels in the clustering 
result is found, and the line segment from the average value of the two long sides of the circumscribed rectangle is taken as the corresponding power line in the result image.

\section{4 Deviation Calculation}

According to the slope, all power line segments are divided into horizontal power line segment and vertical power line segment. The slope of horizontal power line segment is less than 1 , and the slope of vertical power line segment is greater than 1 . The average value of all vertical line segments is taken as the UAV tracking direction of distribution network power line. In order to control the UAV line inspection, the heading deviation and roll deviation should be transmitted to the UAV. So, the deviation should be calculated according to the information of the track line segment in the image.



Fig.3 This is a figure. Schemes follow the same formatting. The direction of UAV distribution network power line tracking. The blue line represents the inspected distribution line. The green line represents the direction of UAV distribution network power line tracking. A and B are the vertex of the tracking line segment. The size of image is width* height.

The heading deviation of UAV $\theta$ is calculated according to the Eq. (3). $\left[u_{A}, v_{A}\right]$ is the pixel coordinate of the higher vertex $\mathrm{A}$ of the tracking line segment in the image. $\left[u_{B}, v_{B}\right]$ is the pixel coordinate of the lower vertex B of the tracking line segment in the image. The vertical line in the center of the image is taken as the benchmark. Therefore, the angle (between the track line segment and the benchmark) on the right side of benchmark is positive, and on the left side of benchmark is negative.

$$
\theta=\arctan \left(u_{A}-u_{B}\right) /\left(v_{B}-v_{A}\right)
$$

The roll deviation of UAV is calculated according to the Eq. (4). We take the image center $\mathrm{O}$ as the benchmark, and the track line segment is positive on the right side of benchmark and negative on the left side of benchmark. The distance of the roll deviation is the distance between $\mathrm{P}$ (intersection point of the straight line where the tracing line segment is located and the horizontal center line of the image) and $\mathrm{O}$. 


$$
\Delta x=u_{A}-\left(\text { height } / 2-v_{A}\right)\left(u_{A}-u_{B}\right) /\left(v_{B}-v_{A}\right)-\text { width } / 2
$$

\section{Results and discussion}

\section{1 Dataset and Evaluation Standard}

In this paper, a distribution power line dataset is provided. The Distribution Power Line Dataset is the small-scale dataset for testing deep learning methods on the distribution line detection task. Existing datasets are all for transmission line. Powerline Image Dataset [27] is a public dataset contains 4000 Infrared and 4000 visible light images without annotated. NAL-RGB Dataset contains 530 power line images captured by UAV, but unpublished. Extracting the distribution power line from the aerial images is one of the most essential parts in distribution power line detection. Meanwhile, there is no public distribution power line dataset to test deep learning methods on the distribution power line detection. Therefore, it is very meaningful to provide a public dataset for distribution power line detection.

To collect data, we controlled the UAV flight manually on distribution power line and recorded videos in many places in Zhejiang Province on different days. 17 videos were collected. One image of every 5 frames was extracted and annotated to construct the dataset. It consists of 4873 images under good and medium weather conditions, of which 3074 are annotated. To achieve good diversity, the dataset contains diverse scene types such as rural, city streets and buildings. Furthermore, the dataset was recorded in diverse season conditions. We divided the dataset into 3 detecting categories, which includes all the challenging scenarios in practical application. We also provide the original high resolution $(480 \mathrm{p})$ video in our dataset. 


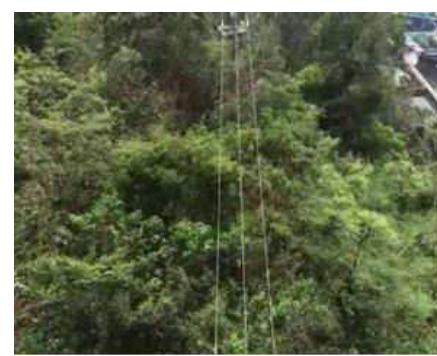

rural

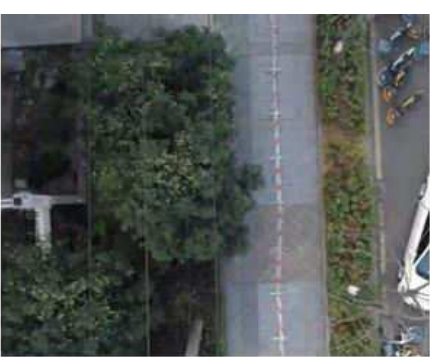

city streets



buildings

\section{Detecting categories}



Fig.4 Proportion of the three detection categories in the dataset.

To generate the ground-truth mask from the images, we use the Labelme image annotator which is publicly available free to coast. The annotation for each image come in a jason format. We manually annotate the distribution power lines along the edge of each distribution power line without distinction. In many cases, the distribution power lines are blocked by tall trees. We annotate the ground-truth even through the middle of distribution power line is occluded partially. However, some distribution power lines, which are almost completely occluded or hard to identify, are not annotated. This way, the network will learn to predict distribution power line location even they are occluded partially.



(a)

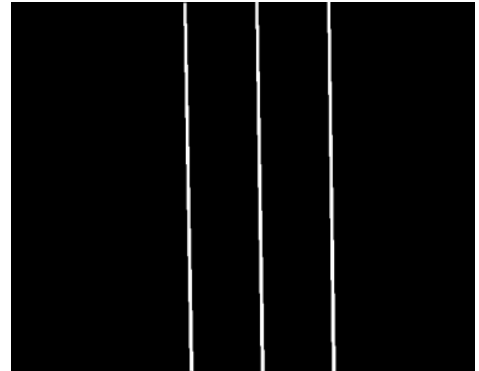

(b)



(c)

Fig.5 An example taken from Distribution Power Line Dataset. (a) Original image taken by UAV, (b) ground-truth label of distribution power line, (c) visualization of distribution power line annotation.

The dataset evaluation standard used for the training of semantic segmentation network are the Jaccard index (Intersection over Union) and the testing speed of network. Jaccard index is used to 
compare the similarity and difference between a finite number of sets. The calculation of it is the ratio of intersection and union of two sets of true value and predicted value.

$$
\mathrm{IoU}=\mathrm{TP} /(\mathrm{TP}+\mathrm{FN}+\mathrm{FP})
$$

where TP refers to the number of present in the image and correctly detected. FP denotes the number of not present in the image but detected, and FN is the number of present in the image but not detected.

In order to evaluate the method proposed, we also provide the test video of the method in the dataset. The video was captured by the rotor UAV in both urban and suburban environments. The frame of every 5 frames in the test video is extracted to calculate the heading deviation and roll deviation of the frame image. The direction of the power line is marked in the extracted image manually. Deviation data for each test video is recorded for evaluating the method proposed.

Due to the heading deviation and roll deviation are calculated as the output of method, the evaluation standard for method includes heading evaluation standard and roll evaluation standard. The average error and standard deviation of heading deviation are the evaluation standard of heading deviation. The smaller the absolute value of the average error and the standard deviation of the error are, the better the method is. The ratio of the number of frames successfully tracked to the number of frames extracted from the video is the evaluation standard of roll deviation. The successful tracking frame is defined with the distance between the heading deviation calculated in a frame and the marked deviation is less than $1 / 2$ of the distance between the two farthest distribution lines. Therefore, the calculated power line trend is in the area of power line. The higher the ratio of the number of frames successfully tracked to the number of frames extracted from the video is, the better the method is.

\section{2 Network Comparison}

In order to verify the effectiveness of network proposed, two groups of experiments were carried out. First, the network was compared with the state-of-the-art network. The network chosen was demonstrated to be the most appropriate for distribution power line detection by combining the accuracy with speed. Second, the experiments of different network structure parameters are carried out on the network structure. The network structure parameters with the best overall effect are obtained.

The proposed network was trained with Adam optimizer. Cross-entropy loss function is used to calculate the training loss. The cross-entropy loss function of pixel coordinate [i, j] is Eq. (6). $p_{i, j}$ stands for the ground-truth label of pixel [i, j]. $q_{i, j}$ stands for the forecast result of pixel [i, j]. The learning rate decreases linearly with training until 0.

$$
L_{i, j}=-\left[p_{i, j} \log q_{i, j}+\left(1-p_{i, j}\right) \log \left(1-q_{i, j}\right)\right]
$$

The training parameters are shown in the Table 1 . The backbone network utilizes the MobileNetV2 pre-training model. All proposed experiments were performed in the PyTorch deep learning framework on GTX GeForce 1080Ti GPU, executed under Linux operating system.

\begin{tabular}{ll}
\hline Parameters & Value \\
\hline Batch size & 8 \\
Epoch & 200 \\
Num classes & 2 \\
Weight decay rate & 0.0005 \\
Momentum rate & 0.9 \\
Initial learning rate & 0.01 \\
\hline
\end{tabular}

Table 1 The training parameters of network. 
The comparison with the state-of-the-art network is shown in the Table 2. The size of all input are $3 * 600 * 480$. The Intersection over Union (IoU) rate can be significantly improved by adding ASPP module to the backbone network. And it will not make the processing speed much slower. Compared with the model with ResNet [28] or Dilated Residual Networks (DRN) [29] as the backbone network, the processing speed is increased by 7-1000 times. Compared with the lane line state-of-the-art network Special CNN (SCNN) [30] and the nested U-Net [31] adopted in [23], the IoU rate and processing speed are significantly improved.

\begin{tabular}{|c|c|c|c|c|}
\hline Network & $\begin{array}{l}\text { IoU } \\
(\%)\end{array}$ & $\begin{array}{c}\text { rate Speed } \\
(\mathrm{ms})\end{array}$ & FLOPS & Params \\
\hline MobileNetV2 + ASPP + DeepLabv3+(Decoder) & 86.07 & 11 & 14.7G & $1.4 \mathrm{M}$ \\
\hline MobileNetV2 + DeepLabv3+(Decoder) & 84.41 & 9 & $14.2 \mathrm{G}$ & $1.2 \mathrm{M}$ \\
\hline ResNet101 + ASPP + DeepLabv3+(Decoder $)$ & 87.71 & 83 & $213.9 \mathrm{G}$ & $46.4 \mathrm{M}$ \\
\hline ResNet50 + ASPP + DeepLabv3+(Decoder $)$ & 86.46 & 71 & $128.5 \mathrm{G}$ & $27.4 \mathrm{M}$ \\
\hline DRN + ASPP + DeepLabv3+(Decoder $)$ & 88.55 & 14667 & $170.1 \mathrm{G}$ & $36.4 \mathrm{M}$ \\
\hline SCNN & 85.76 & 153 & $23.9 \mathrm{G}$ & $20.2 \mathrm{M}$ \\
\hline Nested U-Net & 66.81 & 37 & $150.9 \mathrm{G}$ & $9.0 \mathrm{M}$ \\
\hline
\end{tabular}

Table 2 The IoU rate and processing speed for the different network architecture.

Finally, different output stride and low-level features channels parameters are tested for the network structure. The experimental results show that the overall performance of the network is the best when output stride $=8$ and double expansion of low-level features channels.
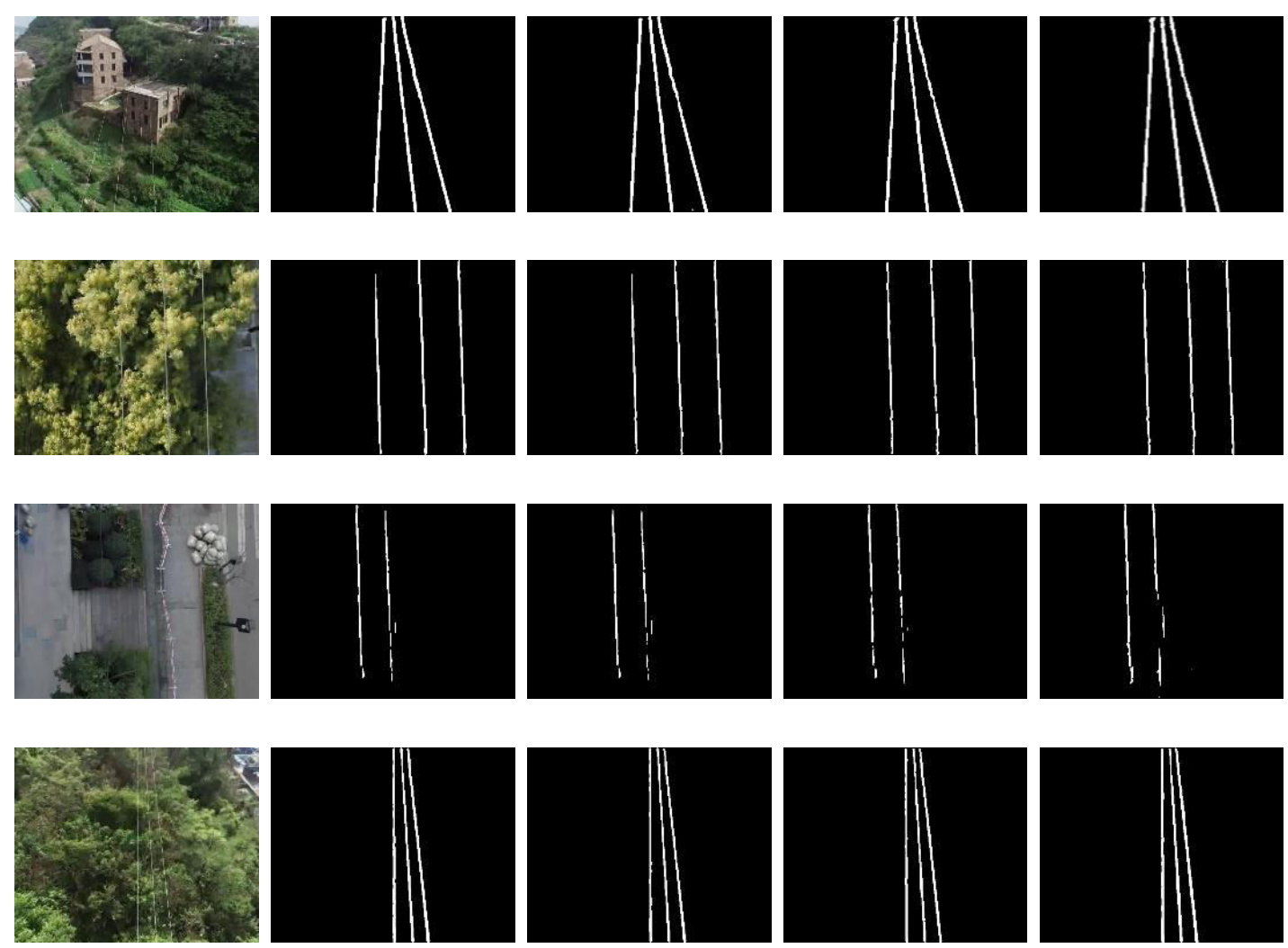

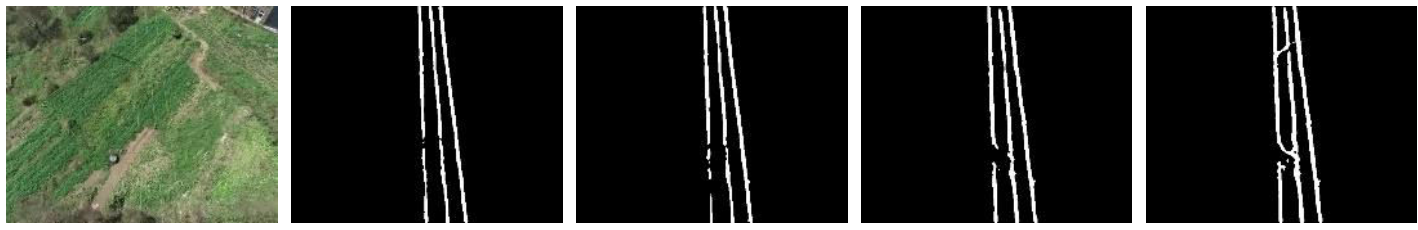

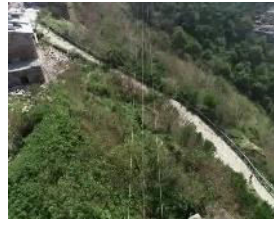

(a)



(b)

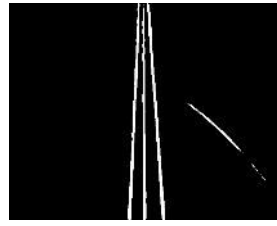

(c)

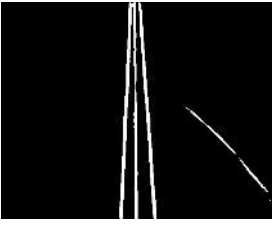

(d)

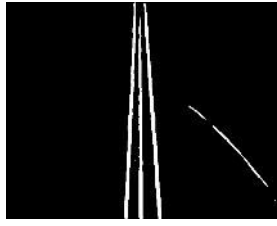

(e)

Fig.6 Visual results obtained using different output stride and low-level features channels parameters of the network structure selected. (a) The input image of deep learning network, (b) the results of output stride $=8$ and double expansion of low-level features channels, (c) the results of output stride $=8$ and double reduction of low-level features channels, (d) the results of output stride $=16$ and double expansion of low-level features channels, (e) the results of output stride $=16$ and double reduction of low-level features channels.

Table 3 The IoU rate and processing speed for different output stride and low-level features channels parameters of the network

\begin{tabular}{llll}
\hline Output stride & Low-level features channels & IoU rate $(\boldsymbol{\%})$ & Speed $(\mathbf{m s})$ \\
\hline 16 & Expand $(2 \times)$ & 85.26 & 10 \\
16 & Reduce $(0.5 \times)$ & 84.72 & 9 \\
$\mathbf{8}$ & Expand $(\mathbf{2 \times})$ & $\mathbf{8 6 . 0 7}$ & $\mathbf{1 1}$ \\
8 & Reduce $(0.5 \times)$ & 85.82 & 10 \\
\hline
\end{tabular}

structure selected.

\section{3 Method Test}

We conduct experiments on test videos in two environments to verify the accuracy of the proposed vision-based UAV distribution line inspection method by using deep learning. In order to get the best output stride and low-level features channels parameter training, the network model trained with different output stride and low-level features channels parameters for each video is tested.
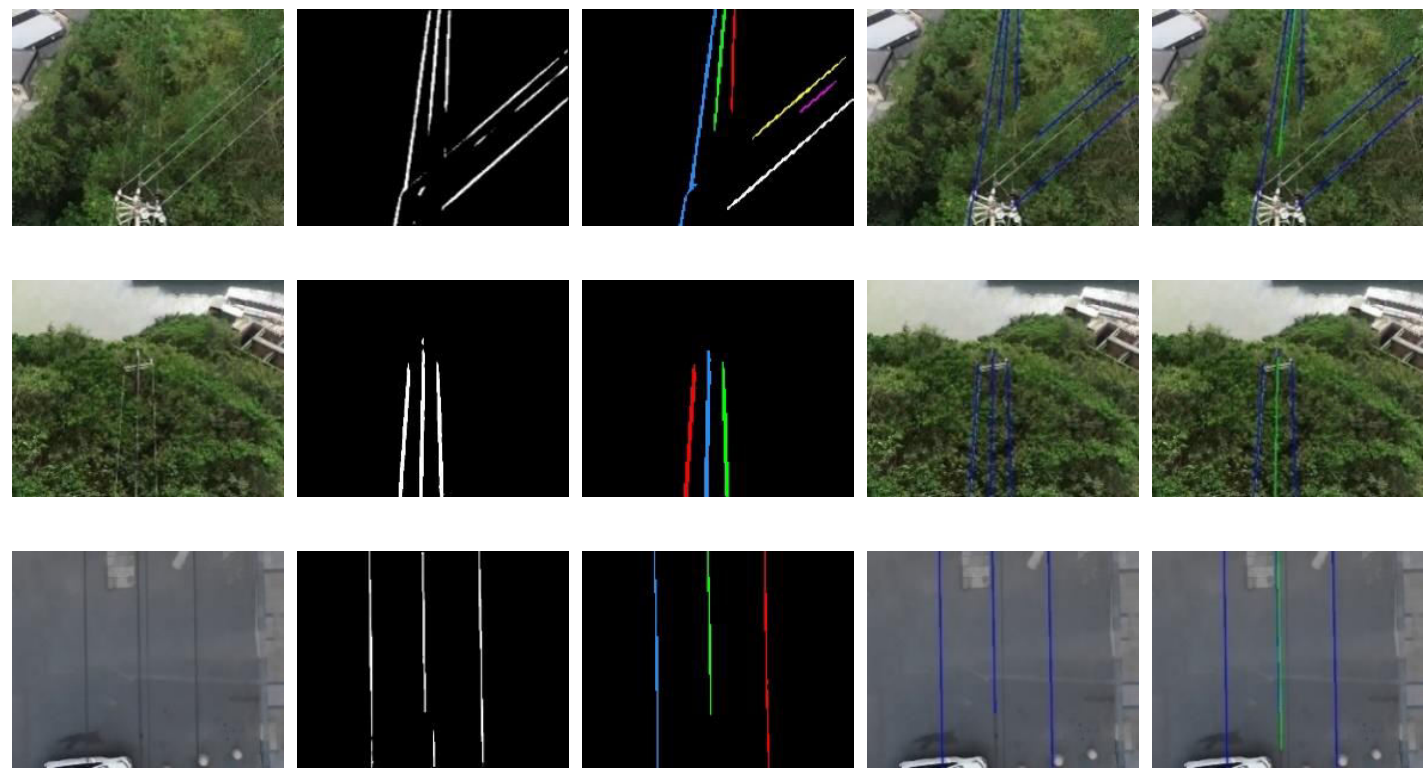




(a)

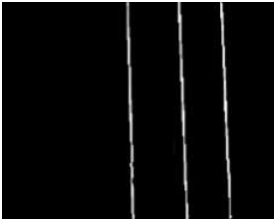

(b)

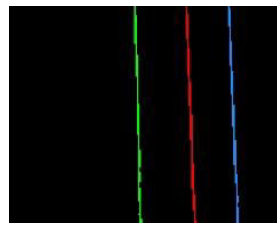

(c)

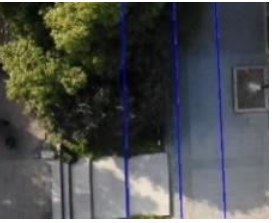

(d)



(e)

Fig.7 Visual results obtained using the method proposed. (a) The visible light image taken from UAV, (b) the prediction results of deep learning, (c) the results of sampling clustering, (d) the results of detected distribution line, (e) the results of the direction of UAV distribution network power line tracking.

The results of the two test videos of the method are shown in Table 4. The method can reach a inspection speed of nearly $77 \mathrm{~ms}$ per frame. The heading and roll accuracy calculated by the method is the best when output stride $=8$ and the low-level features channels are double expanded. In the video 1 test results, the range of heading deviation error is $\left(-2.67^{\circ}, 3.21^{\circ}\right)$, and the tracking rate is $99.33 \%$. In the video 2 test results, the range of heading deviation error is $\left(-1.52^{\circ}, 1.36^{\circ}\right)$, and the tracking rate is $100 \%$. From the experimental results, the proposed vision-based UAV distribution line inspection method by using deep learning can quickly and accurately realize distribution line inspection.

Table 4 This is a table. Tables should be placed in the main text near to the first time they are cited.

\begin{tabular}{llll}
\hline Video & Network parameters & Heading deviation error $\left({ }^{\circ}\right)$ & Tracking rate \\
\hline 1 & output stride $=8$, Expand $(2 \times)$ & average $=0.2711, \sigma=0.9882$ & $99.33 \%$ \\
1 & output stride $=8$, Reduce $(0.5 \times)$ & average $=0.5948, \sigma=1.1586$ & $97 \%$ \\
1 & output stride $=16$, Expand $(2 \times)$ & average $=-0.1944, \sigma=1.2578$ & $96.33 \%$ \\
1 & output stride $=16$, Reduce $(0.5 \times)$ & average $=0.0866, \sigma=1.4607$ & $96.67 \%$ \\
\hline 2 & output stride $=8$, Expand $(2 \times)$ & average $=-0.0838, \sigma=0.4853$ & $100 \%$ \\
2 & output stride $=8$, Reduce $(0.5 \times)$ & average $=-0.0408, \sigma=0.5092$ & $100 \%$ \\
2 & output stride $=16$, Expand $(2 \times)$ & average $=-0.1094, \sigma=0.4151$ & $100 \%$ \\
2 & output stride $=16$, Reduce $(0.5 \times)$ & average $=-0.1027, \sigma=0.4576$ & $100 \%$ \\
\hline
\end{tabular}



(a) 


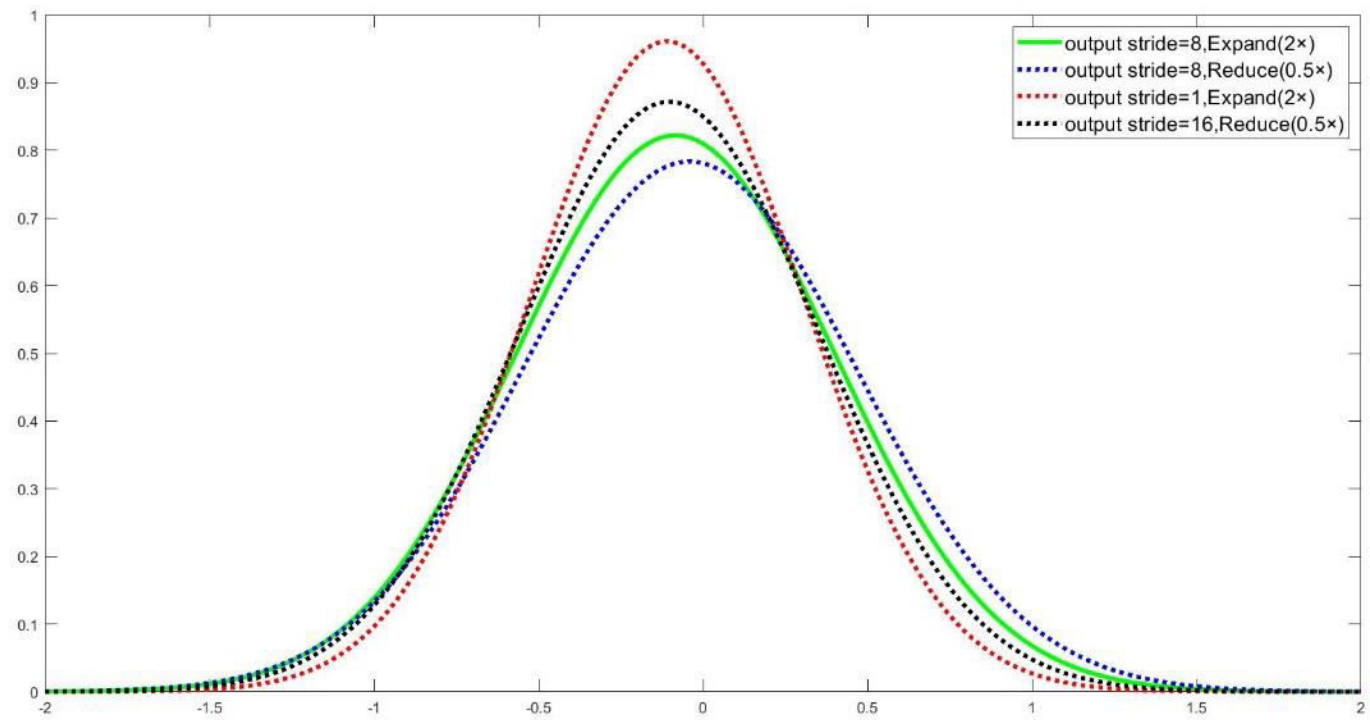

(b)

Fig.8 Standard normal distribution of the heading deviation error. (a) Result from test video 1, (b) result from test video 2.

\section{Conclusion}

In this paper, a vision-based UAV distribution line inspection method using deep learning is proposed for UAV distribution network line inspection. Firstly, the deep learning network with encoder-decoder structure is used to quickly and accurately predict the distribution line area in the picture. Then, for the network prediction results, effective image processing operations and sampling clustering are used to remove the interference and determine the distribution line in the image. Finally, the UAV tracking direction of distribution network power line is calculated according to the detected distribution line. The dataset of deep learning method for testing distribution line detection task is proposed, and the test video and evaluation criteria are provided. In the experiment, the network comparison and method test results show that the method proposed in this paper is effective, fast and accurate for UAV distribution line inspection. The IOU rate of the network model in the proposed method on the dataset can reach $86.08 \%$, and the processing speed can reach $11 \mathrm{~ms}$. The tracking rate of the proposed method on the test video can be guaranteed to be more than $99 \%$.

In the future work, we will focus on the decision-making calculation of UAV patrol line under the condition of multi-line intersection and strengthen the application of the method in project practice.

\section{Abbreviations}

UAV: Unmanned aerial vehicle

CNN: Convolutional Neural Networks

R-CNN: Region with Convolutional Neural Networks features

FCN: Fully convolutional network

ASPP: Atrous spatial pyramid pooling

DBSCAN: Density based spatial clustering of applications with noise

IoU: Intersection over union

TP: True positive 
FP: False positive

FN: False negative

FLOPS: Floating-point operations per second

Params: Parameters

\section{Declarations}

\section{Ethics approval and consent to participate}

Not applicable.

\section{Consent for publication}

Not applicable.

\section{Availability of data and materials}

The datasets generated during the current study are available from the corresponding author on reasonable request.

\section{Competing interests}

The authors declare that they have no competing interests.

\section{Funding}

Not applicable.

\section{Authors' contributions}

X. C. and J.Z. contributed to the conception and methodology of the study; software, J. Z. ; X.C., J.Z. and H.W. performed the experiment and validation; H.W. and M. X. contributed to the formal analysis; X. C., J. Z. and M. X. contributed to the investigation of the study; J.Z. and H.W. contributed to the software and data curation; X.C. and J.Z. performed the data analyses and wrote the manuscript; X. C. and J.Z. performed the data visualized; E. Z. and M. X. contributed to the supervision; project administration, E. Z performed the project administration. All authors have read and agreed to the published version of the manuscript.

\section{Acknowledgements}

Not applicable.

\section{Author details}

${ }^{1}$ Unmanned System Application Technology Research Institute, China Jiliang University, Hangzhou 310018, China. ${ }^{2}$ Hangzhou Mission Intelligent Technology Company Limited, Hangzhou 310018, China.

\section{References}

1. D. Li, X. Wang, The Future Application of Transmission Line Automatic Monitoring and Deep Learning Technology Based on Vision, in: 2019 IEEE 4th International Conference 
on Cloud Computing and Big Data Analysis, pp. 131-137. https://doi.org/10.1109/ICCCBDA.2019.8725702

2. V. N. Nguyen, R. Jenssen, D. Roverso, Automatic autonomous vision-based power line inspection: A review of current status and the potential role of deep learning, International Journal of Electrical Power \& Energy Systems. 2018, vol. 99, pp. 107-120. https://doi.org/10.1016/i.ijepes.2017.12.016

3. J. Zhang, H. Wang, Y. Hu, Design of Intelligent Inspection System for Distribution Network Based on Circular Multi-Agent Structure, in: 2019 IOP Conference Series Earth and Environmental Science. https://doi.org/10.1088/1755-1315/218/1/012136

4. T. Santos, PLineD: Vision-based power lines detection for Unmanned Aerial Vehicles, in: 2017 IEEE International Conference on Autonomous Robot Systems and Competitions, pp. 253-259. https://doi.org/10.1109/ICARSC.2017.7964084

5. B. Chen, X. Miao, Distribution Line Pole Detection and Counting Based on YOLO Using UAV Inspection Line Video, Journal of Electrical Engineering and Technology. 2020, vol.15, pp. 441-448. https://doi.org/10.1007/s42835-019-00230-w

6. J. Katrasnik, F. Pernus and B. Likar, A Survey of Mobile Robots for Distribution Power Line Inspection, in: IEEE Transactions on Power Delivery. 2010, vol. 25, pp. 485-493. https://doi.org/10.1109/TPWRD.2009.2035427

7. L. Yang, J. Fan, Y. Liu, A Review on State-of-the-Art Power Line Inspection Techniques, in: IEEE Transactions on Instrumentation and Measurement. 2020 vol. 69, pp. 9350-9365. https://doi.org/10.1109/TIM.2020.3031194

8. P. B. Castellucci, L.C. Lucca, Pole and Crossarm Identification in Distribution Power Line Images, 2013 Latin American Robotics Symposium and Competition, pp. 2-7. https://doi.org/10.1109/LARS.2013.48

9. H. Sharma, R. Bhujade, V. Adithya, Vision-based detection of power distribution lines in complex remote surroundings, 2014 Twentieth National Conference on Communications, pp. 1-6. https://doi.org/10.1109/NCC.2014.6811357

10. L. F. Luque-Vega, B. Castillo-Toledo, A. Loukianov, Power line inspection via an unmanned aerial system based on the quadrotor helicopter, in: MELECON 2014 - 2014 17th IEEE Mediterranean Electrotechnical Conference, pp. 393-397. https://doi.org/10.1109/MELCON.2014.6820566

11. K. Takaya, H. Ohta, V. Kroumov, Development of UAV System for Autonomous Power Line Inspection, in: 2019 23rd International Conference on System Theory, Control and Computing, pp. 762-767. https://doi.org/10.1109/ICSTCC.2019.8885596

12. A. Cerón, I.F. Mondragón B., F. Prieto, Towards Visual Based Navigation with Power Line Detection, in: Advances in Visual Computing, vol. 8887, pp 827-836. https://doi.org/10.1007/978-3-319-14249-4 79

13. Z. Li, Y. Liu, R. Hayward, J. Zhang, Knowledge-based power line detection for UAV surveillance and inspection systems, in: 2008 23rd International Conference Image and Vision Computing New Zealand, pp. 1-6. https://doi.org/10.1109/IVCNZ.2008.4762118

14. L. Zhao, X. Wang, Power Line Extraction from Aerial Images Using Object-Based Markov Random Field with Anisotropic Weighted Penalty, in: IEEE Journal of Selected Topics in Applied Earth Observations and Remote Sensing, vol. 8(2015), pp. 1924-1935. https://doi.org/10.1109/ACCESS.2019.2939025

15. I.E. Nordeng, A. Hasan, D. Olsen, DEBC Detection with Deep Learning, in: Image Analysis. SCIA 2017. Lecture Notes in Computer Science, vol. 10269, pp. 248-259. https://doi.org/10.1007/978-3-319-59126-1 21

16. M. Lan, Y. Zhang, L. Zhang, Defect detection from UAV images based on region-based CNNs, in: 2018 IEEE International Conference on Data Mining Workshops, pp. 385-390. https://doi.org/10.1109/ICDMW.2018.00063

17. J. Tan, Automatic Insulator Detection for Power Line Using Aerial Images Powered by Convolutional Neural Networks, Journal of Physics: Conference Series. 2021, vol. 1748. https://doi.org/10.1088/1742-6596/1748/4/042012 
18. W. Chang, G. Yang, E. Li, Toward a Cluttered Environment for Learning-Based Multi-Scale Overhead Ground Wire Recognition, Neural Process Lett. 2018, 48, pp. 17891800. https://doi.org/10.1007/s11063-018-9799-3

19. F. Fambrini, Y. Iano, D. G. Caetano, GPU Cuda JSEG Segmentation Algorithm associated with Deep Learning Classifier for Electrical Network Images Identification, Procedia Computer Science. 2018, vol.126, pp. 557-565. https://doi.org/10.1016/j.procs.2018.07.290

20. S. Ren, K. He, R. Girshick, Faster R-CNN: Towards Real-Time Object Detection with Region Proposal Networks, in: IEEE Transactions on Pattern Analysis and Machine Intelligence, vol. 39, no. 6, pp. 1137-1149. https://doi.org/10.1109/TPAMI.2016.2577031

21. E. Shelhamer, J. Long, T. Darrell, Fully Convolutional Networks for Semantic Segmentation, in: IEEE Transactions on Pattern Analysis and Machine Intelligence, vol. 39, no. 4, pp. 640-651. https://doi.org/10.1109/TPAMI.2016.2572683

22. X. Hui, J. Bian, X. Zhao, Vision-based autonomous navigation approach for unmanned aerial vehicle transmission-line inspection, International Journal of Advanced Robotic Systems. 2018, vol.15. https://doi.org/10.1177/1729881417752821

23. S. Saurav, P. Gidde, S. Singh, Power Line Segmentation in Aerial Images Using Convolutional Neural Networks, in: Pattern Recognition and Machine Intelligence, Lecture Notes in Computer Science, vol. 11941(2019), pp. 623-632. https://doi.org/10.1007/978-3-030-34869-4 68

24. M. Sandler, A. Howard, M. Zhu, MobileNetV2: Inverted Residuals and Linear Bottlenecks, in: 2018 IEEE/CVF Conference on Computer Vision and Pattern Recognition, pp. 4510-4520. https://doi.org/10.1109/CVPR.2018.00474

25. L. Chen, Y. Zhu, G. Papandreou, Encoder-Decoder with Atrous Separable Convolution for Semantic Image Segmentation, Computer Vision - ECCV 2018, pp. 833-851. https://doi.org/10.1007/978-3-030-01234-2 49

26. M. Ester, H. P. Kriegel, J. Sander, A Density-Based Algorithm for Discovering Clusters in Large Spatial Data-bases with Noise, Proceedings of 2nd International Conference on Knowledge Discovery and Data Mining, pp. 226-231.

27. Ö.E. Yetgin, Ö.N. Gerek, Powerline image dataset (infrared-IR and visible light-VL), Mendeley Data, v7 (2017). http://dx.doi.org/10.17632/n6wrv4ry6v.7

28. K. He, X. Zhang, S. Ren and J. Sun, Deep Residual Learning for Image Recognition, in: 2016 IEEE Conference on Computer Vision and Pattern Recognition, pp. 770-778. http://dx.doi.org/10.1109/CVPR.2016.90

29. F. Yu, V. Koltun and T. Funkhouser, Dilated Residual Networks, 2017 IEEE Conference on Computer Vision and Pattern Recognition, pp. 636-644. http://dx.doi.org/10.1109/CVPR.2017.75

30. X. Pan, J. Shi, P. Luo, Spatial as deep: Spatial CNN for traffic scene understanding, in: 32nd AAAI Conference on Artificial Intelligence, pp. 7276-7283.

31. Z. Zhou, M.M. Rahman Siddiquee, N. Tajbakhsh, UNet++: A Nested U-Net Architecture for Medical Image Segmentation, in: Deep Learning in Medical Image Analysis and Multimodal Learning for Clinical Decision Support, DLMIA 2018, ML-CDS 2018. LNCS, vol. 11045, pp. 3-11. https://doi.org/10.1007/978-3-030-00889-5 1 